\title{
High Transmission Wave-Guide Wire Network Made by Self-Assembly
}

\author{
Stefano Salvatore ${ }^{1}$, Silvia Vignolini ${ }^{1}$, Julian Philpott, Morgan Stefik $^{2}$, \\ Ulrich Wiesner ${ }^{2}$, Jeremy J. Baumberg ${ }^{1}$, Ullrich Steiner ${ }^{1}$ \\ ${ }^{1}$ Cavendish Laboratory, Department of Physics, University of Cambridge, \\ J. J. Thomson Avenue, Cambridge CB3 0HE, UK. \\ ${ }^{2}$ Department of Materials Science \& Engineering, Cornell University, Ithaca, New York 14853, USA
}

July 15, 2014

Optical metamaterials have recently received increasing attention, enabling fascinating and sought-after properties and functionalities such as negative refractive indices [1, 2], superlensing $[1,3]$ and cloaking devices $[4,5]$. While there are now a large number of metamaterials that are active at microwave, terahertz and infrared frequencies [4,6-11], metamaterials for visible wavelengths are problematic because of their difficulty in fabrication and their high losses, which are the main obstacles towards optical applications [12]. Reducing these losses to enable high optical transmission is therefore the key to design of effective and functional optical metamaterials.

Optical metamaterials that are based on 3D sub-micrometer periodic lattices rely on the propagation of surface-plasmon polaritons across the network structure. These electron plasma oscillations are damped in the two materials adjacent to the interface. While the dissipation in one of these phases (air) is negligible, substantial losses occur in the metal that supports the plasmon resonance. The only way to solve this conundrum is by reducing the amount of metal at the plasmonic interface, similar to the utilisation of coaxial cables and wave-guides for high frequency signal transduction.

Here, we present an implementation of this approach by creating a three dimensional selfassembled metamaterial consisting of a hollow-wire-network that shows exceptionally high transmission and reduced absorption. This work is based on recent theoretical [13, 14] and experimental $[15,16]$ studies employing the chiral gyroid morphology as a model system for 3D optical metamaterials. A negative refractive index at optical wavelengths has been predicted for this topology [17].

Our approach is based on the self-assembly of block copolymers [18], consisting of two or more covalently tethered linear chains. Self-assembly in these systems is driven by microphase separation that balances the reduction of the free energy of mixing with the creation of unfavourable interfaces and chain stretching [19]. This results in a large range of periodic 3D architectures controlled by the number of polymer blocks and their relative molecular weight $[20]$.

In this study, a polyisoprene-block-polystyrene-block-polyethylene oxide (ISO) terpolymer was used that assembles into two interpenetrating, chemically distinct gyroid networks (isoprene and ethylene oxide), which are embedded in a majority styrene phase. The ISO has an overall molecular weight of $33 \mathrm{~kg} \mathrm{~mol}^{-1}$ with relative volume fractions of $30 \%$ (isoprene), $53 \%$ (styrene) and $17 \%$ (ethylene oxide) that self-assembles upon annealing into a double gyroid morphology with a unit cell size of $35 \mathrm{~nm}$. The polyisoprene phase is selective degraded by UV irradiation, 


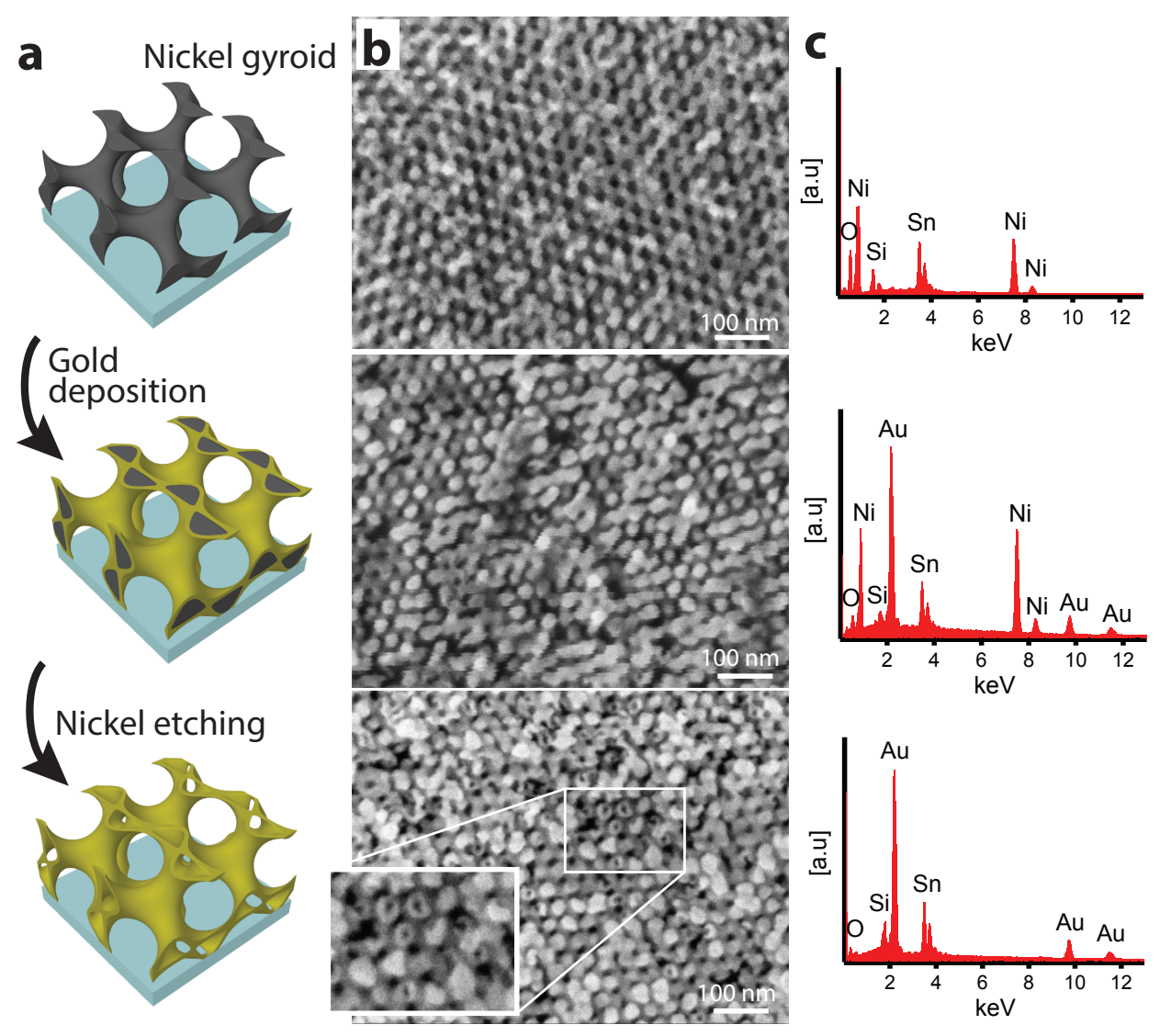

Figure 1: Hollow gyroid manufacture process; left, schematic; middle, SEM micrographs; right, EDX measurements. (a) Nickel gyroid is fabricated as described previously [15]. (b) Coating of the $\mathrm{Ni}$ gyroid with a thin layer of gold by electrodeposition. (c) Selective etching of the $\mathrm{Ni}$ by $\mathrm{FeCl}_{3}$ reveals a hollow $\mathrm{Au}$ gyroid. The SEM images in (b) show a thickening of the struts compared to (a) due to the Au deposition while the struts are hollow in (c) after Ni etching. This is corroborated by the EDX data showing the complete removal of $\mathrm{Ni}$ in (c).

producing a porous template that is back-filled by nickel electrodeposition from a conducting substrate. The remaining polymers are subsequently removed by plasma etching, resulting in a free-standing $\mathrm{Ni}$ single gyroid (Fig. 1a, top). This nickel structure is then used as the working electrode for electrodeposition of gold, which deposits homogeneously around the $\mathrm{Ni}$ struts (Fig. 1a, middle). In the final step, the inner nickel core is etched away by immersion in $\mathrm{FeCl}_{3}$, resulting in a hollow gyroid morphology (Fig. 1a, bottom). SEM images of the three manufacturing stages and their corresponding energy dispersive x-ray spectroscopy (EDX) results are shown in Fig. 1.

The SEM images in Fig. 1a,b show the the homogeneous deposition of $\mathrm{Au}$ around the Ni struts. Despite this conformity of the gold coating, the nickel core is readily etched away by immersion in $\mathrm{FeCl}_{3}$, as verified by SEM and EDX in Fig. 1c. This indicates that local defects and interstitial diffusion at the Au grain boundaries of the gold coating allows $\mathrm{FeCl}_{3}$ access to the Ni-core during the etching process, without destabilising the surrounding Au-shell.

The amount of deposited gold was measured by EDX [21] relative to the nickel content before removal by etching. Since the amount of Ni per unit cell (fill fraction, $f$ ) is determined by the the isoprene volume fraction $(\sim 30 \%)$ of the ISO polymer, the EDX elemental Au:Ni ratio in Fig. 1b directly determines the Au fill fraction. The EDX result in Fig. 1c confirms the complete removal of the nickel core.

Reflection and transmission spectra of the coaxial hollow gyroid are compared to Au gyroids, 

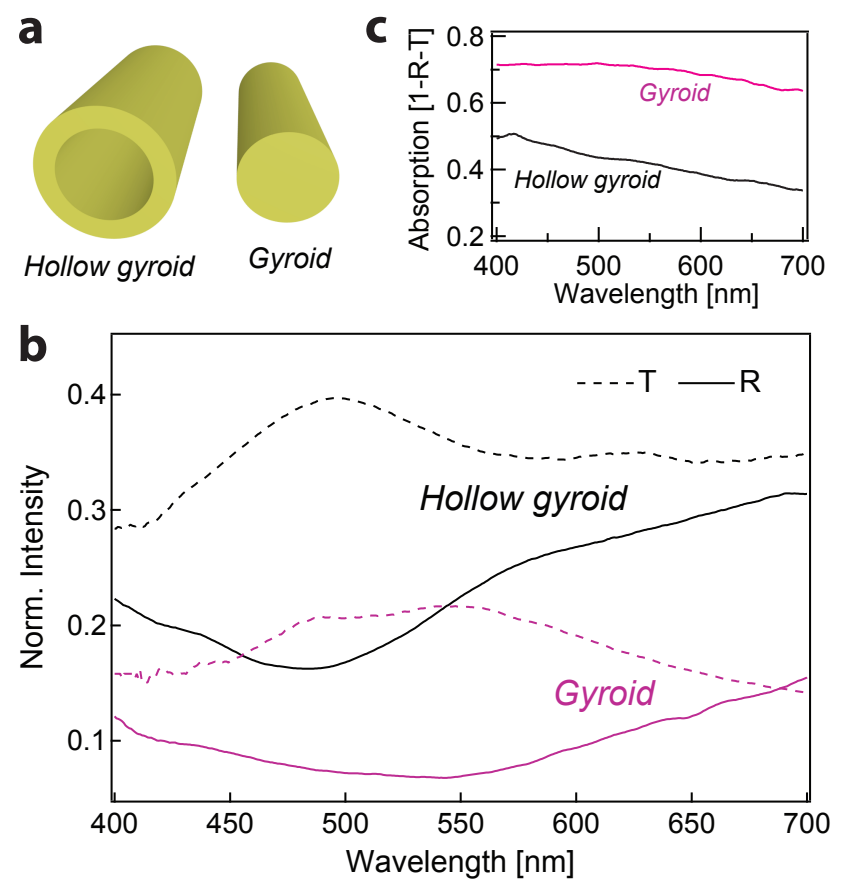

Figure 2: (a) Schematic of the strut morphology of the coaxial hollow gyroid and full gyroid. (b) Reflection and transmission spectra of the hollow gyroid compared with the full gyroid. (c) Absorption spectra calculated from the reflection and transmission intensities. Films thickness: $300 \mathrm{~nm}, f \approx 30 \%$.

in which $\mathrm{Au}$ was directly deposited into the voided polymer matrix, similar to Fig. 1a, resulting in a "full" gyroid with similar overall filling fraction. Despite nearly identical amounts of gold in the gyroid unit cell, the reflection and transmission intensities of the hollow Au gyroid are enhanced by a factor of 2 with respect to the full Au gyroid, as shown in Fig. 1.

As demonstrated before, the full Au gyroid metamaterial is characterised by a reduced gold plasma frequency [15] that results in a red-shift of the plasma edge. Increasing the full gyroid filling fraction shifts the plasma edge to shorter wavelengths, approaching the value of solid $\mathrm{Au}$ for $f \rightarrow 1$. This is mirrored by the spectra in Fig. 2b where the inflection point of the reflection spectrum of the hollow gyroid (around $530 \mathrm{~nm}$ ) lies at a shorter wavelength compared to the full gyroid. This is consistent with the earlier result, since for constant $f$, the coaxial hollow gyroid has an increased outer strut radius compared to the full gyroid.

The measurement of both the transmission and reflection spectra allow us (in the absence of scattering which is small in these samples) to calculate the optical absorption shown in Fig. 2c. For constant $f$, absorption in the $300 \mathrm{~nm}$ thick hollow gyroid film is reduced by a factor of two compared to the full gyroid, yielding, according to this theory, a longer effective plasma wavelength. For constant $f$ and lattice symmetry, the lower optical dissipation of Fig. 2c is clearly linked to the detailed Au morphology.

One of the advantages of our sample manufacture technique is the facile control of the fill-fraction; the wall width can simply be varied by adjusting the Au electrodeposition time. Gold filling fractions, as measured by EDX, with a Au to Ni ratio between $10 \%$ and $30 \%$, were produced in this way. Figure 3 establishes a clear trend: reducing $f$ from $30 \%$ to $10 \%$ increases transmission at $\approx 500 \mathrm{~nm}$ from 0.4 for $f \approx 30 \%$ to $\approx 0.48$ and $\approx 0.58$ for $f=20 \%$ and $f=10 \%$, respectively. Measuring the values of transmission and reflection at $500 \mathrm{~nm}$ yields absorptions of $0.44,0.42$ and 0.36 for $f$-values of $30 \%, 20 \%$, and $10 \%$, respectively.

These results are remarkable in several ways. Starting from the same self-assembled gyroid, 


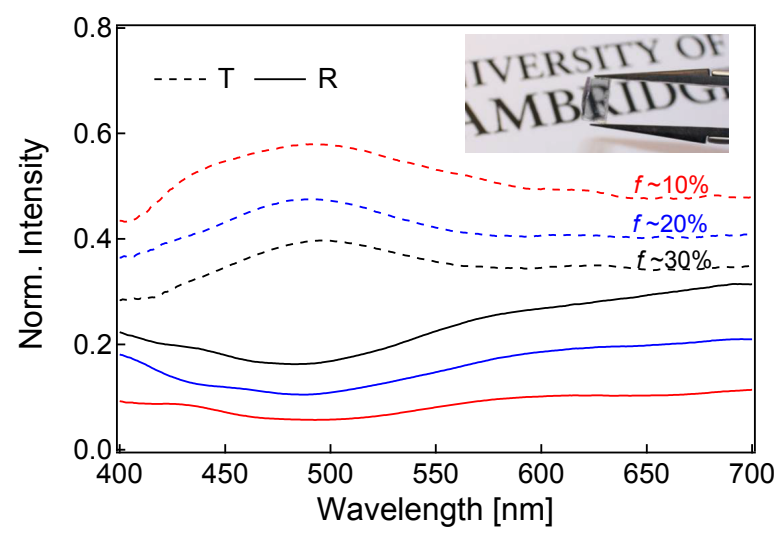

Figure 3: Reflection and transmission spectra of hollow gyroids for filling fractions of 30\%, 20\% and $10 \%$. The filling fraction was measured by EDX analysis before nickel core removal. The inset shows a photograph of the hollow gyroid with $f=10 \%$. Film thickness: $300 \mathrm{~nm}$.

careful tuning of Au composition increases transmission across a $300 \mathrm{~nm}$ film by nearly a factor of 3 to an unprecedented value of $\sim 60 \%$, which is perhaps best visualised by the image in the inset of Fig. 3. Starting from a Ni-strut width of $\approx 13 \mathrm{~nm}$, the Au-shell of the $f=10 \%$ hollow gyroid has a wall width of less than $1 \mathrm{~nm}$. The structural stability of such a 3D hollow network structure is truly astounding.

While the detailed interplay of optical and plasmonic modes in the full and hollow gyroids requires extensive numerical simulations [13, 14], the data of Figs. 2 and 3 allow a qualitative analysis. The amount of transmitted light across a film of constant thickness depends on three parameters: (1) the number of transmission modes per unit area, (2) the efficiency of in- and out-coupling into (and out of) these transmission modes, and (3) the optical absorption per mode. Since the hollow struts provide a wave-guide type structure, additional plasmon modes propagating insides the struts can contribute to the overall transmission. Optical absorption is clearly reduced, by about a factor of 2 when comparing the full and hollow gyroids. Since the skin-depth of Au varies between 20 and $50 \mathrm{~nm}$ for optical frequencies, all conduction-band electrons participate in the surface plasmon resonances that propagate along the gyroid struts. Per mode, the dissipation depends on the overall thickness of the material (i.e. the number of electrons that participate in the oscillation and their displacement amplitude), which is clearly borne out by the data.

The changes in propagating modes and absorption alone do not however fully account for the data in Fig. 3. The morphology in these three samples is very similar, and it is unlikely that the number of propagating modes differs significantly between them. While changes in absorption from 0.44 to 0.36 are seen when going from $f=30 \%$ to $f=10 \%$ which corroborates the argument above, it accounts for only approx $1 / 2$ of the $18 \%$ absolute increase in transmission. In the absence of scattering, the difference has to be attributed to the reflection channel: the in-coupling of light into the $f=10 \%$ hollow gyroid is about $10 \%$ more efficient compared to the $f=30 \%$ gyroid.

Careful comparison of the 4 datasets in Figs. 2 and 3 therefore suggests that an interplay of all three mechanisms are responsible for the extraordinary transmission of light across the $300 \mathrm{~nm}$ thick Au metamaterial. Hollow components within metamaterials can thus cause changes to the input coupling, propagation loss, and add additional propagating modes.

In conclusion we have demonstrated a substantial transmission enhancement across an optical metamaterial based on a block copolymer self-assembled morphology. Optical transmission was increased by a factor of nearly 3 when replacing the full Au struts with hollow wave-guide 
morphologies. The overall transmission was shown to depend sensitively on the wall-thickness of the hollow struts, which was demonstrated down to $1 \mathrm{~nm}$. Our data indicates that the dramatic transmission increase arises from an interplay of three mechanisms, (1) the additional number of modes propagating through the wave-guide structure, (2) increased efficiency of light in-coupling, and (3) a reduction of dissipation by decreasing the Au-volume experienced in plasmon mode propagation to values much below the Au skin-depth.

These findings are likely to be useful for the design of 3D metamaterials and can pave the way to applications requiring low-loss optical metamaterials.

\section{Acknowledgements}

We acknowledge the EPSRC EP/G060649/1, the Doctoral Training Centre NanoDTC EP/..., and ERC LINASS 320503 for funding.

\section{References}

[1] Pendry, J. Contemporary Physics 2004, 45, 191-202.

[2] Smith, D.; Pendry, J.; Wiltshire, M. Science 2004, 305, 788-792.

[3] Pendry, B. J. B.; Smith, D. R. Scientific American 2006, July, 60-67.

[4] Smith, D. R.; Padilla, W. J.; Vier, D.; Nemat-Nasser, S. C.; Schultz, S. Physical review letters 2000, 84, 4184.

[5] Schurig, D.; Mock, J.; Justice, B.; Cummer, S.; Pendry, J.; Starr, A.; Smith, D. Science 2006, 314, 977-980.

[6] Smith, D.; Padilla, W.; Vier, D.; Nemat-Nasser, S.; Schultz, S. Negative permeability from split ring resonator arrays. 2000.

[7] Yen, T.-J.; Padilla, W.; Fang, N.; Vier, D.; Smith, D.; Pendry, J.; Basov, D.; Zhang, X. Science 2004, 303, 1494-1496.

[8] Katsarakis, N.; Konstantinidis, G.; Kostopoulos, A.; Penciu, R.; Gundogdu, T.; Kafesaki, M.; Economou, E.; Koschny, T.; Soukoulis, C. Optics Letters 2005, 30, 1348-1350.

[9] Linden, S.; Enkrich, C.; Wegener, M.; Zhou, J.; Koschny, T.; Soukoulis, C. M. Science (New York, N.Y.) 2004, 306, 1351-3.

[10] Zhang, S.; Fan, W.; Panoiu, N.; Malloy, K.; Osgood, R.; Brueck, S. Optics express 2006, 14, 6778-6787.

[11] Burgos, S. P.; de Waele, R.; Polman, A.; Atwater, H. A. Nature Materials 2010, 9, 407412.

[12] Smith, D. Science 2010, 32\%, 138-139.

[13] Demetriadou, A.; Oh, S. S.; Wuestner, S.; Hess, O. New Journal of Physics 2012, 14, DOI: $10.1088 / 1367-2630 / 14 / 8 / 083032$.

[14] Oh, S. S.; Demetriadou, A.; Wuestner, S.; Hess, O. Advanced Materials 2012, Doi: 10.1002/adma.201202788. 
[15] Vignolini, S.; Yufa, N. a.; Cunha, P. S.; Guldin, S.; Rushkin, I.; Stefik, M.; Hur, K.; Wiesner, U.; Baumberg, J. J.; Steiner, U. Advanced materials (Deerfield Beach, Fla.) 2012, 24, OP23-7.

[16] Salvatore, S.; Demetriadou, A.; Vignolini, S.; Oh, S. S.; Wuestner, S.; Yufa, N. a.; Stefik, M.; Wiesner, U.; Baumberg, J. J.; Hess, O.; Steiner, U. Advanced materials (Deerfield Beach, Fla.) 2013, 25, 2713-6.

[17] Hur, K.; Francescato, Y.; Giannini, V.; Maier, S. a.; Hennig, R. G.; Wiesner, U. Angewandte Chemie (International ed. in English) 2011, 50, 11985-9.

[18] others, et al. The physics of block copolymers; Oxford University Press New York, 1998; Vol. 19.

[19] Flory, P. J. Journal of Chemical Physics 1942, 10, 51-61.

[20] Bates, F. S.; Fredrickson, G. H. Physics Today 1999, 32-38.

[21] Goldstein, J.; Newbury, D. E.; Joy, D. C.; Lyman, C. E.; Echlin, P.; Lifshin, E.; Sawyer, L.; Michael, J. R. Scanning electron microscopy and X-ray microanalysis; Springer, 2003. 